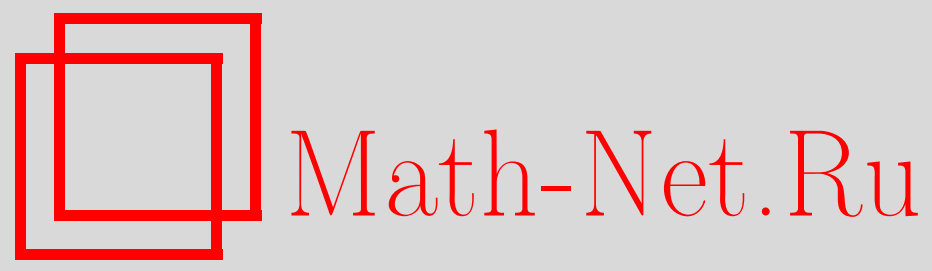

A. 3. Насыров, Об обходе автоматами лабиринтов в $n$ мерном пространстве, Дискрет. матем., 2000, том 12, выпуск 4, 121-137

DOI: https://doi.org/10.4213/dm352

Использование Общероссийского математического портала Math-Net.Ru подразумевает, что вы прочитали и согласны с пользовательским соглашением http: //www . mathnet.ru/rus/agreement

Параметры загрузки:

IP: 3.85 .7 .115

26 апреля 2023 г., 17:59:50

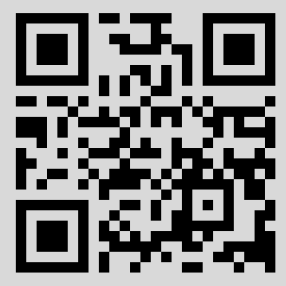




\title{
Об обходе автоматами лабиринтов в $n$-мерном пространстве
}

\author{
(c) 2000 г. A. 3. Насыров
}

\begin{abstract}
Рассматривается проблема обхода автоматами пространственных лабиринтов. Доказано, что существует автомат, оставляющий в вершинах лабиринта одну нестираемую метку (краску) и обходящий произвольный $n$-мерный прямоугольный лабиринт.
\end{abstract}

\section{1. Введение}

Проблема обхода плоских лабиринтов конечными автоматами была поставлена K. Шенноном в начале шестидесятых годов. Л. Будахом было доказано [1], что не существует конечного автомата, который обходил бы произвольный шахматный лабиринт.

Поиски положительного решения проблемы обхода лабиринтов автоматами велись в двух направлениях. Первое направление связано с рассмотрением более узких классов лабиринтов $[4,5]$, а второе - с усилением возможностей автоматов. Одно из возможных усилений - рассмотрение коллективов автоматов. В [6] показано, что коллектив, состоящий из двух автоматов или одного автомата и двух камней, обходит произвольный конечный плоский лабиринт. Но уже в трехмерном пространстве существует ловушка для любого коллектива автоматов [7]. Более полный обзор результатов, полученных в этих направлениях, можно найти в [3].

В качестве другого усиления можно рассмотреть автоматы, оставляющие метки в вершинах лабиринта. Было доказано [8], что существует автомат с одной краской, обходящий произвольный плоский прямоугольный лабиринт. В данной работе этот результат обобщен на случай $n$-мерных прямоугольных лабиринтов.

\section{2. Постановка задачи и формулировка основного результата}

Основные понятия из теории автоматов, используемые в этой статье, совпадают с принятыми в [2] и здесь не определяются.

Пусть $X$ - некоторое множество. Через $P_{0}(X)$ обозначим множество всех непустых подмножеств множества $X$, а через $|X|$ - мощность множества $X$. Пусть $\left\{X_{\alpha}\right\}_{\alpha \in A}-$ некоторое индексированное семейство множеств. Тогда для любого $\alpha \in$ $A$ через $\operatorname{Pr}_{\alpha}$ обозначим отображение проецирования произведения $\prod_{\beta \in A} X_{\beta}$ на его 
сомножитель $X_{\alpha}$ с номером $\alpha$. Если множество индексов $A$ конечно, то будем полагать, что $A=\{1, \ldots,|A|\}$.

Пусть $L=(V, \Gamma)$ - связный ориентированный граф, не имеющий петель и кратных дуг, $V$ - множество вершин, $\Gamma$ - множество дуг графа $L$. Граф $L=(V, \Gamma)$ называется симметрическим, если для любой дуги $\left(v_{1} ; v_{2}\right) \in \Gamma$ дуга $\left(v_{2} ; v_{1}\right)$ также входит в $\Gamma$.

Если всем дугам графа $L=(V, \Gamma)$ приписаны метки из некоторого алфавита $\Sigma$ таким образом, что разным дугам, исходящим из одной и той же вершины, а также разным дугам, входящим в одну и ту же вершину, приписаны разные метки, то этот нагруженный граф назовем лабиринтом. Метку дуги $\gamma \in \Gamma$ обозначим через $|\gamma|$. Лабиринт $L$ с выделенной (начальной) вершиной $v_{0}$ называется инициальным, обозначим его через $L_{v_{0}}$. Для любой вершины $v$ лабиринта $L=(V, \Gamma)$ пусть $d_{\text {in }}(v, L)$ - множество меток дуг, входящих в вершину $v$ и $d_{\text {out }}(v, L)$ - множество меток дуг, выходящих из вершины $v$, причем, когда ясно, о каком лабиринте идет речь, будем писать $d_{\text {in }}(v)$ и $d_{\text {out }}(v)$. В дальнейшем рассматриваются только конечные лабиринты (множества вершин и дуг конечны).

Обозначим через $\mathscr{E}^{n}$ множество $\left\{e_{1}, \ldots, e_{n}\right\}$ базисных единичных векторов $n$ мерного евклидова пространства $\mathbf{R}^{n}$, а через $\mathscr{E}^{n}-$ множество

$$
\left\{e_{1}, \ldots, e_{n}, \bar{e}_{1}, \ldots, \bar{e}_{n}\right\}
$$

где $\bar{e}_{i}=-e_{i}, i=1, \ldots, n$.

Пусть $M, N \in \mathbf{R}^{n}, M \neq N$ и $\overline{M N}=\alpha_{1} e_{1}+\ldots+\alpha_{n} e_{n}$. Будем говорить, что отрезок $\overline{M N}$ идет в направлении $e_{i}$, если $\alpha_{i}>0$ и $\alpha_{j}=0$, и в направлении $\bar{e}_{i}$, если $\alpha_{i}<0$ и $\alpha_{j}=0$ для всех $j \neq i$. Множество $T$ отрезков в $\mathbf{R}^{n}$ называется конфигурацией, если любые два отрезка из $T$ имеют не более одной общей точки, причем, если она есть, то является концевой для обоих отрезков.

Лабиринт $L=(V, \Gamma)$ с множеством меток дуг $\overline{\mathscr{E}}^{n}$ называется $n$-мерным прямоугольным лабиринтом, если $L-$ связный симметрический граф, $V \subset \mathbf{R}^{n}$, для любой дуги $(u ; v) \in \Gamma$ отрезок $\overline{u v}$ идет в направлении $|(u ; v)|$ и множество отрезков $T=\{\overline{u v} \mid(u ; v) \in \Gamma\}$ является конфигурацией.

Обозначим класс всех $n$-мерных прямоугольных лабиринтов через $\mathbf{L}^{n}$.

Инициальный автомат $\mathfrak{A}_{q_{0}}=\left(A, Q, B, \varphi, \psi, q_{0}\right)$ называется автоматом с одной краской, если $A=E_{2} \times P_{0}\left(\mathscr{E}^{n}\right)-$ входной алфавит, $B=E_{2} \times \overline{\mathscr{E}}^{n}-$ выходной алфавит, а функция выходов $\psi$ такова, что для любых $q \in Q, a \in A$

$$
\operatorname{Pr}_{2}(\psi(q, a)) \in \operatorname{Pr}_{2}(a)
$$

и если $\operatorname{Pr}_{1}(a)=1$, то $\operatorname{Pr}_{1}(\psi(q, a))=1$.

Пусть $\mathfrak{A}_{q_{0}}-$ некоторый инициальный автомат с одной краской, $L_{v_{0}}-$ инициальный $n$-мерный прямоугольный лабиринт, вершины которого могут быть помечены символами из $E_{2}$. Поведение автомата $\mathfrak{A}_{q_{0}}$ в лабиринте $L_{v_{0}}$ интерпретируем следующим образом. В каждый момент времени автомат обозревает какую-то из вершин лабиринта. Пусть автомат находится в состоянии $q$ и обозревает вершину $v$ лабиринта. Тогда входной буквой автомата в этот момент времени будет пара $a=\left(\omega, d_{\text {out }}(v)\right)$, где $\omega$ - метка вершины $v$. Если $\psi(q, a)=\left(\omega^{\prime}, e\right)$, то автомат ставит метку $\omega^{\prime}$ в вершину $v$, переходит в состояние $\varphi(q, a)$ и перемещается в вершину, в которую из $v$ ведет дуга с меткой $е$. При этом, если $\omega=1$ (вершина $v$ окрашена), то и $\omega^{\prime}=1$. В начальный момент автомат $\mathfrak{A}_{q_{0}}$ находится в состоянии $q_{0}$ и обозревает вершину $v_{0}$. 
Формально поведение автомата в лабиринте определяется через понятие конфигурации. Конфигурацией автомата $\mathfrak{A}_{q_{0}}=\left(A, Q, B, \varphi, \psi, q_{0}\right)$ с одной краской в $n$ мерном прямоугольном лабиринте $L_{v_{0}}=\left(V, \Gamma, v_{0}\right)$ называется тройка $K=(q, v, \omega)$, где $q \in Q, v \in V$, а $\omega: V \rightarrow\{0 ; 1\}$ - разметка лабиринта, то есть $\omega(v)=1$, если метка вершины $v$ равна 1 , и $\omega(v)=0$, если метка $v$ равна 0. Говорят, что конфигурация $\left(q^{\prime}, v^{\prime}, \omega^{\prime}\right)$ следует за конфигурацией $(q, v, \omega)$ и обозначают $(q, v, \omega) \vdash\left(q^{\prime}, v^{\prime}, \omega^{\prime}\right)$, если

$$
\begin{aligned}
& q^{\prime}=\varphi(q, a),\left(v ; v^{\prime}\right) \in \Gamma, \quad\left|\left(v ; v^{\prime}\right)\right|=\operatorname{Pr}_{2}(\psi(q, a)), \\
& \omega^{\prime}(u)= \begin{cases}\operatorname{Pr}_{1}(\psi(q, a)), & \text { если } u=v, \\
\omega(u), & \text { если } u \neq v,\end{cases}
\end{aligned}
$$

где $a=\left(\omega(v), d_{\text {out }}(v)\right)$. Поведением автомата $\mathfrak{A}_{q_{0}}$ в лабиринте $L_{v_{0}}$ называется последовательность $\pi\left(\mathfrak{A}_{q_{0}}, L_{v_{0}}\right)$ конфигураций $K_{0}, K_{1}, K_{2}, \ldots$ таких, что $K_{0}=\left(q_{0}, v_{0}, \omega_{0}\right)$, где $\omega_{0}=0$, и $K_{i} \vdash K_{i+1}$ для всех $i=0,1,2, \ldots$

Через $\pi_{i}\left(\mathfrak{A}_{q_{0}}, L_{v_{0}}\right)$ обозначим $i$-й элемент последовательности $\pi\left(\mathfrak{A}_{q_{0}}, L_{v_{0}}\right)$ и рассмотрим

$$
\operatorname{Int}\left(\mathfrak{A}_{q_{0}}, L_{v_{0}}\right)=\bigcup_{i=0}^{\infty} \operatorname{Pr}_{2} \pi_{i}\left(\mathfrak{A}_{q_{0}}, L_{v_{0}}\right)
$$

- множество всех вершин, которые обходит автомат $\mathfrak{A}_{q_{0}}$ в лабиринте $L_{v_{0}}$.

Инициальный автомат $\mathfrak{A}_{q_{0}}$ обходит инициальный лабиринт $L_{v_{0}}=\left(V, \Gamma, v_{0}\right)$, если $\operatorname{Int}\left(\mathfrak{A}_{q_{0}}, L_{v_{0}}\right)=V$.

Если в множестве $Q$ состояний автомата $\mathfrak{A}_{q_{0}}$ выделено подмножество $Q_{F}$ заключительных состояний, то будем считать, что автомат прекращает обход при переходе в одно из заключительных состояний. В этом случае последовательность $\pi\left(\mathfrak{A}_{q_{0}}, L_{v_{0}}\right)$ может быть конечной.

Будем говорить, что автомат $\mathfrak{A}_{q_{0}}$ обходит лабиринт $L_{v_{0}}$ и останавливается после обхода, если $\operatorname{Int}\left(\mathfrak{A}_{q_{0}}, L_{v_{0}}\right)=V$ и $\pi\left(\mathfrak{A}_{q_{0}}, L_{v_{0}}\right)$ конечна.

Теорема 1. Существует автомат с одной краской, обходящий произволъный $n$ мерный прямоуголъный лабиринт и останавливающийся после обхода.

\section{3. $n$-лабиринты}

Лабиринт $L=(V, \Gamma)$ с множеством меток дуг $\mathscr{E}^{n}$ называется $n$-лабиринтом, если $L-$ сильносвязный ориентированный граф и $d_{\text {in }}(v)=d_{\text {out }}(v)$ для любой вершины $v \in V$.

Определим отображение $F: P_{0}\left(\overline{\mathscr{E}}^{n}\right) \rightarrow P_{0}\left(\mathscr{E}^{n}\right)$ ' таким образом, что для любых $M \in P_{0}\left(\overline{\mathscr{E}}^{n}\right)$ и $i=1, \ldots, n$

$$
e_{i} \in F(M) \Longleftrightarrow\left\{e_{i}, \bar{e}_{i}\right\} \cap M \neq \varnothing
$$

Пусть $L=(V, \Gamma)$ - произвольный $n$-мерный прямоугольный лабиринт. Множество его дуг $\Gamma$ можно представить в виде объединения попарно непересекающихся множеств $\Gamma_{i}, 1 \leqslant i \leqslant n$, где

$$
\Gamma_{i}=\left\{\gamma \in \Gamma|| \gamma \mid \in\left\{e_{i}, \bar{e}_{i}\right\}\right\}
$$


Рассмотрим граф $L_{i}=\left(V, \Gamma_{i}\right)$. Каждая компонента связности графа $L_{i}$ либо представляет собой подграф графа $L$ с упорядоченным множеством вершин $\left\{v_{1}, \ldots, v_{k}\right\}$ и множеством дуг

$$
\left\{\left(v_{1} ; v_{2}\right), \ldots,\left(v_{k-1} ; v_{k}\right),\left(v_{k} ; v_{k-1}\right), \ldots,\left(v_{2} ; v_{1}\right)\right\}
$$

причем

$$
\left|\left(v_{j} ; v_{j+1}\right)\right|=e_{i}, \quad\left|\left(v_{j+1} ; v_{j}\right)\right|=\bar{e}_{i}
$$

для любого $j=1, \ldots, k-1$, либо является изолированной вершиной. Такой подграф графа $L$ будем называть $e_{i}$-коридором в лабиринте $L$, вершину $v_{1}$ - начальной, а $v_{k}$ - конечной вершиной этого $e_{i}$-коридора.

Рассмотрим лабиринт $\tilde{L}=(V, \tilde{\Gamma})$, получающийся из лабиринта $L$ заменой для каждого $i$ всех $e_{i}$-коридоров на ориентированные циклы по следующему правилу. В каждом $e_{i}$-коридоре удаляются все дуги с меткой $\bar{e}_{i}$ и добавляется дуга с меткой $e_{i}$, идущая из конечной вершины этого $e_{i}$-коридора в начальную. Легко показать, что лабиринт $\tilde{L}$ является $n$-лабиринтом, причем

$$
d_{\text {out }}(v, \tilde{L})=F\left(d_{\text {out }}(v, L)\right) .
$$

Полученный таким образом лабиринт $\tilde{L}$ будем называть $n$-лабиринтом, порожденным $n$-мерным прямоугольным лабиринтом $L$, а добавленные дуги, соединяющие конечные и начальные вершины коридоров, будем, для краткости, называть длинными дугами. У лабиринтов $L$ и $\tilde{L}$ множества вершин совпадают. Значит, обход лабиринта $\tilde{L}$ является одновременно обходом лабиринта $L$. Позднее будет сформулирован алгоритм обхода именно для порожденных $n$-лабиринтов. Поэтому, для получения автомата, реализующего этот алгоритм в $n$-мерных прямоугольных лабиринтах, необходимо построить автоматы, которые моделировали бы перемещения по дугам $n$-лабиринта.

Для удобства задания автоматов расширим выходные алфавиты автоматов с одной краской символом $\Lambda$, который будет означать, что автомат остается в той же вершине, не изменяя ее метки. Измениться, при этом, может лишь состояние автомата. Заметим, что такое расширение выходного алфавита не увеличивает возможностей автомата при обходе лабиринтов. Более того, можно, изменив функции переходов $\varphi$ и выходов $\psi$, исключить использование символа $\Lambda$, не изменяя, при этом, множества пройденных вершин. Например, если $\varphi(q, a)=q^{\prime}$ и $\psi(q, a)=\Lambda$, то можно задать $\varphi$ и $\psi$ для пары $(q, a)$, полагая

$$
\varphi(q, a)=\varphi\left(q^{\prime}, a\right), \quad \psi(q, a)=\psi\left(q^{\prime}, a\right) .
$$

Рассмотрим автоматы с одной краской $\mathfrak{B}_{+}^{i}$ и $\mathfrak{B}_{-}^{i}, i=1, \ldots, n$, диаграммы Мура которых изображены на рис. 1. На диаграммах использованы следующие обозначения. Рядом с каждой стрелкой, соответствующей переходу между состояниями, стоит дробь. В числителе дроби записаны условия, которым должна удовлетворять входная пара $(\omega, X) \in A$ для этого перехода, а в знаменателе - получающийся на выходе автомата символ из алфавита $B \cup\{\Lambda\}$.

Лемма 1. Пустъ $L$ - произвольный $n$-мерный прямоуголъный лабиринт, $\tilde{L}-$ порожденный им $n$-лабиринт, $v$ - вериина лабиринта $L$, причем $e_{i} \in d_{\text {out }}(v, \tilde{L})$. Eсли автомат $\mathfrak{B}_{+}^{i}$ поместить в вериину $v$ в начальном состоянии $q_{0}$, то он переместится в вершину, в которую из $v$ ведет дуга с меткой $e_{i}$ лабиринта $\tilde{L}$, и перейдет 


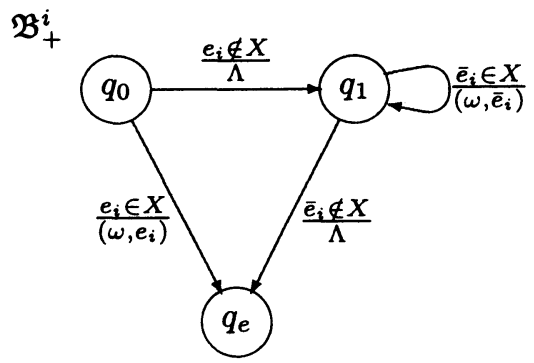

$\mathfrak{B}_{-}^{i}$

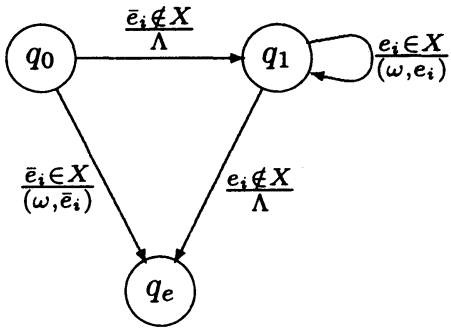

Рис. 1.

в заключителъное состояние $q_{e}$. Если автомат $\mathfrak{B}_{-}^{i}$ поместитъ в вериину $v$ в началъном состоянии $q_{0}$, то он переместится в вериину, из которой в $v$ ведет дуга с меткой $e_{i}$ лабиринта $\tilde{L}$, и перейдет в заключителъное состояние $q_{e}$.

Доказательство. Если $v$ - конечная вершина $e_{i}$-коридора, то $e_{i} \notin d_{\text {out }}(v, L)$ и в $\tilde{L}$ дуга с меткой $e_{i}$ ведет из $v$ в начальную вершину этого $e_{i}$-коридора. В этом случае автомат $\mathfrak{B}_{+}^{i}$, согласно диаграмме, перейдет в состояние $q_{1}$ и будет оставаться в нем, перемещаясь по дугам с меткой $\bar{e}_{i}$, пока это будет возможно, то есть пока не попадет в начальную вершину $e_{i}$-коридора, после чего перейдет в состояние $q_{e}$. Если $v$ не является конечной вершиной $e_{i}$-коридора, то дуга с меткой $e_{i}$ лабиринта $\tilde{L}$ является одновременно и дугой лабиринта $L$, а автомат $\mathfrak{B}_{+}^{i}$, совершив переход по этой дуге, перейдет из состояния $q_{0}$ в состояние $q_{e}$. Автомат $\mathfrak{B}_{-}^{i}$ рассматривается аналогично.

Замечание 1. Если автоматы $\mathfrak{B}_{+}^{i}$ и $\mathfrak{B}_{-}^{i}$ поместить в вершину, из которой в лабиринте $\tilde{L}$ не выходит дуга с меткой $e_{i}$, то они перейдут в заключительное состояние, оставаясь в исходной вершине.

Пусть $\tilde{L}=(V, \tilde{\Gamma})-$ произвольный $n$-лабиринт. Рассмотрим дугу $\gamma \in \tilde{\Gamma}$. Пусть $\gamma=$ $\left(v_{1}, v_{0}\right)$ и $|\gamma|=e_{i}$. Тогда можно построить последовательность вершин $v_{0}, v_{1}, v_{2}, \ldots$ такую, что $\left(v_{j}, v_{j-1}\right) \in \tilde{\Gamma}$ и $\left|\left(v_{j}, v_{j-1}\right)\right|=e_{i}$ для всех $j \in \mathbf{N}$. Действительно, пусть уже найдены вершины $v_{0}, v_{1} \ldots v_{k}$, обладающие этим свойством. Так как $d_{\text {in }}\left(v_{k}\right)=$ $d_{\text {out }}\left(v_{k}\right)$ и $\left|\left(v_{k}, v_{k-1}\right)\right|=e_{i}$, найдется вершина $v_{k+1}$ такая, что $\left|\left(v_{k+1}, v_{k}\right)\right|=e_{i}$. Множество вершин $V$ конечно, поэтому, в последовательности $v_{0}, v_{1}, \ldots$ появятся повторяющиеся вершины. Первой такой вершиной может быть только $v_{0}$, так как из вершины может выходить только одна дуга с меткой $e_{i}$. Значит, дуга $\gamma$ входит в ориентированный цикл, составленный из дуг с метками $e_{i}$. Такой цикл будем называть $e_{i}$-циклом в $n$-лабиринте $\tilde{L}$.

Упорядочим элементы множества $\left\{e_{1}, \ldots, e_{n}\right\}$ так, что

$$
e_{1}<e_{2}<\ldots<e_{n}
$$

Этот порядок естественным образом переносится на множество дуг, выходящих из произвольной вершины $v$-лабиринта, а именно, большей из двух выходящих дуг будет та, у которой метка больше. Таким же образом можно упорядочить и входящие в $v$ дуги. Обозначим через $\gamma_{\max }(v)$ максимальную входящую в вершину $v$ дугу, а ее метку обозначим

$$
e_{\max }(v)=\max _{e \in d_{\text {in }}(v)} e
$$


Число ind $(v)$ такое, что $e_{\text {ind }(v)}=e_{\max }(v)$, будем называть индексом вершины $v$, a последовательность вершин $u_{0}, u_{1}, u_{2}, \ldots$ такую, что $u_{0}=v$,

$$
\left(u_{i+1}, u_{i}\right)=\gamma_{\max }\left(u_{i}\right)
$$

для всех $i \in \mathbf{N}$, назовем обратной последовательностью для вершины $v$.

Предложение 1. Если $\left\{u_{i}\right\}_{i=0}^{\infty}-$ обратная последователъностъ для вершинъ $v$, то для любых $i \leqslant j$ выполнено неравенство $\operatorname{ind}\left(u_{i}\right) \leqslant \operatorname{ind}\left(u_{j}\right)$.

Доказательство. Пусть $i \in \mathbf{N}$. Так как $d_{\text {in }}\left(u_{i}\right)=d_{\text {out }}\left(u_{i}\right)$, максимальные метки входящих и выходящих из $v$ дуг совпадают. Значит, метка дуги

$$
\left(u_{i}, u_{i-1}\right)=\gamma_{\max }\left(u_{i-1}\right)
$$

не превосходит метки максимальной входящей в $u_{i}$ дуги $\gamma_{\max }\left(u_{i}\right)$. Поэтому ind $\left(u_{i-1}\right) \leqslant$ $\operatorname{ind}\left(u_{i}\right)$.

Рассмотрим граф $G$ с множеством вершин $V$ и множеством дуг

$$
\Gamma_{\max }=\left\{\gamma_{\max }(v) \mid v \in V\right\}
$$

Из предложения 1 следует, что если $(u, v) \in \Gamma_{\max }$, то ind $(u) \geqslant \operatorname{ind}(v)$. Пусть

$$
\left\{G_{i}=\left(V_{i}, \Gamma_{i}\right)\right\}_{i=1}^{m}
$$

- множество компонент связности графа $G$. Заметим, что если $v \in V_{i}$, то и все вершины из обратной последовательности для $v$ входят в $V_{i}$.

Предложение 2. Для любого $i$ граф $G_{i}=\left(V_{i}, \Gamma_{i}\right)$ представляет собой ориентированный цикл, $x$ которому присоединены ориентированные от корня деревъя, $c$ корнями в вершинах цикла. Если $e_{k}$ - махсимальная из меток дуг графа $G_{i}$, то дуги с меткой $e_{k}$ образуют цикл в $G_{i}$.

Доказательство. Первое утверждение легко следует из того, что в каждую вершину графа $G_{i}$ входит ровно одна дуга. Индексы вершин из $V_{i}$ не могут превосходить $k$, так как в граф $G_{i}$ вместе с произвольной вершиной $v \in V_{i}$ входит и дуга $\gamma_{\max }(v)$. Пусть $(u, v) \in \Gamma_{i}$ и $|(u, v)|=e_{k}$. Тогда ind $(v)=k$. Рассмотрим обратную последовательность $u_{0}=v, u_{1}, u_{2}, \ldots$ для вершины $v$. По предложению 1 , индексы вершин $u_{1}, u_{2}, \ldots$ больше или равны индексу вершины $u_{0}$, но, с другой стороны, их индексы не превосходят $k$. Значит, индекс любой вершины из этой последовательности равен $k$, то есть $\left|\left(u_{j}, u_{j-1}\right)\right|=e_{k}$ для любого $j \in \mathbf{N}$. Из конечности графа $G_{i}$ следует, что в этой последовательности встретится вершина $u_{0}=v$, то есть дуги с метками $e_{k}$ образуют цикл в $G_{i}$.

Если $\tilde{L}$ порожден $n$-мерным прямоугольным лабиринтом $L=(V, \Gamma)$, то $e_{k}$-циклам в $\tilde{L}$ будут соответствовать $e_{k}$-коридоры в лабиринте $L$. Конечную вершину такого коридора для цикла из компоненты $G_{i}$ будем называть максимальной в множестве $V_{i}$.

Исходя из структуры графа $G_{i}, i=1, \ldots, m$, можно задать циклический обход его вершин. В каждой вершине ориентированного цикла обходим дерево с корнем в этой вершине, после чего переходим к следующей вершине цикла. Обход дерева 


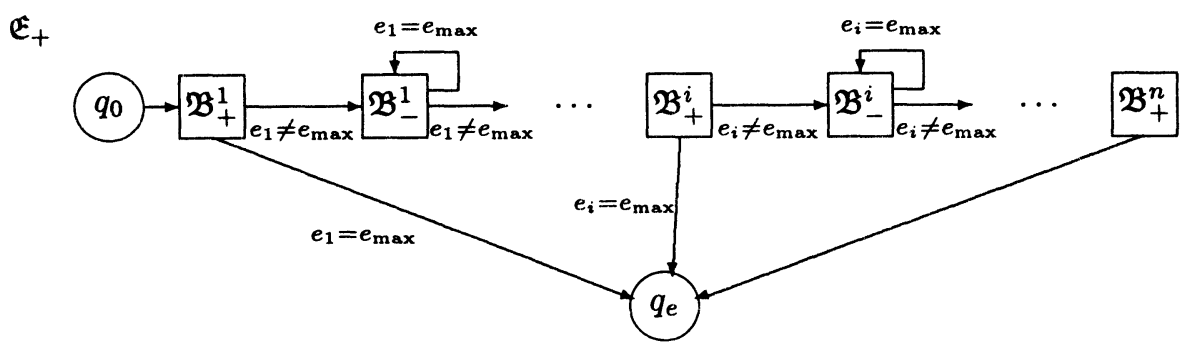

Pис. 2.

производится по следующему правилу. В каждой вершине для продолжения обхода выбирается минимальная из еще не пройденных выходящих дуг. В случаях, когда или вершина является висячей, или все выходящие дуги уже пройдены, возвращаемся по входящим дугам в ближайшую вершину, в которой еще есть непройденные выходящие дуги, и из нее продолжаем обход. Когда все вершины дерева будут пройдены, возвращаемся в корень и переходим к следующей вершине цикла. Учитывая, что дуги в цикле имеют наибольшие метки среди дуг из $\Gamma_{i}$, порядок обхода вершин из $V_{i}$ может быть описан так. Будем обозначать через $\eta(v)$ вершину, следующую за $v$ по обходу. Если вершина $v$ имеет выходящие из нее дуги, то $\eta(v)$ - вершина, в которую ведет минимальная выходящая из $v$ дуга. Пусть $v-$ висячая вершина. Тогда в обратной последовательности $u_{0}, u_{1}, u_{2}, \ldots$ для вершины $v$ ищется вершина $u_{j}$ с минимальным номером, имеющая выходящие дуги с метками большими, чем у дуги $\left(u_{j}, u_{j-1}\right)$. В этом случае, $\eta(v)$ - вершина, в которую ведет минимальная среди выходящих из $u_{j}$ дуг, метки которых больше, чем $\left|\left(u_{j}, u_{j-1}\right)\right|$. Таким образом, задана циклическая перестановка $\eta$ на множестве $V_{i}$.

Определив таким же образом $\eta(v)$ для всех компонент $G_{i}$, получим перестановку $\eta$ на множестве $V$ вершин $n$-лабиринта $\tilde{L}$, причем $\eta$ - циклическая на каждом из множеств $V_{i}$. Разным $n$-лабиринтам будут соответствовать разные перестановки $\eta$. Поэтому, при необходимости указать, что для построения перестановки $\eta$ использовался $n$-лабиринт $\tilde{L}$, будем писать $\eta_{\tilde{L}}$.

Построим теперь автоматы, которые будут осуществлять перестановки $\eta$ и $\eta^{-1}$. Автоматы будем задавать с помощью схем, элементами которых будут ранее заданные автоматы. При этом все входящие в схему автоматы будут иметь начальное состояние и множество заключительных состояний. Стрелка на схеме, ведущая от одного автомата к другому, указывает на то, что из заключительных состояний первого автомата возможен переход в начальное состояние второго автомата, а рядом со стрелкой перечислены заключительные состояния и условия, которым должна удовлетворять входная пара $(\omega, X) \in A$ для такого перехода. В этой ситуации будем говорить, что первый автомат передает управление второму. Если у первого автомата одно заключительное состояние, то оно не будет указываться.

Рассмотрим автомат с одной краской $\mathfrak{E}_{+}$, который задается схемой, изображенной на рис. 2. Через $e_{\max }$ обозначена максимальная метка из множества $F(X)$.

Лемма 2. Автомат $\mathfrak{E}_{+}$, будучи помещеннъм в началъном состоянии $q_{0}$ в произвольную вериину $v$ п-мерного прямоугольного лабиринта $L$, переместится в вершину $\eta(v)$ и перейдет в заключительное состояние $q_{e}$. 


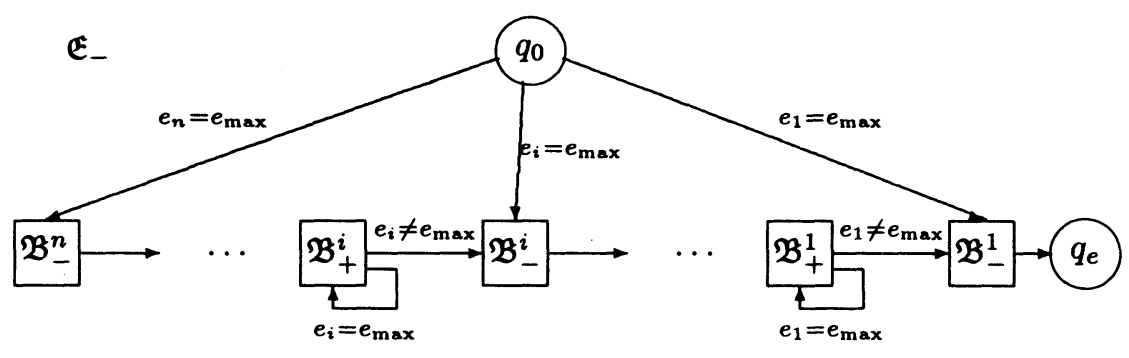

Pис. 3.

Доказательство. Пусть $\tilde{L}-n$-лабиринт, порожденный $L$. Выше было описано правило, как перейти из вершины $v$ в вершину $\eta(v)$, двигаясь по дугам из множества $\Gamma_{\max }$. Автомат $\mathcal{E}_{+}$работает следующим образом. Сперва управление передается автомату $\mathfrak{B}_{+}^{1}$. Если в лабиринте $\tilde{L}$ из $v$ выходит дуга с меткой $e_{1}$, то в результате работы $\mathfrak{B}_{+}^{1}$ произойдет перемещение в вершину, в которую ведет эта дуга. Если эта дуга из множества $\Gamma_{\max }$, то есть $e_{1}=e_{\max }$, то вершина $\eta(v)$ достигнута и автомат $\mathfrak{E}_{+}$ перейдет в заключительное состояние $q_{e}$. Если $e_{1} \neq e_{\max }$, то управление передается автомату $\mathfrak{B}_{-}^{1}$ и автомат $\mathfrak{E}_{+}$возвращается в вершину $v$. Если дуга с меткой $e_{1}$ не является максимальной выходящей дугой из $v$, то управление передается автомату $\mathfrak{B}_{+}^{2}$. Заметим, что если в лабиринте $\tilde{L}$ не было выходящей из $v$ дуги с меткой $e_{1}$, то работа автоматов $\mathfrak{B}_{+}^{1}$ и $\mathfrak{B}_{-}^{1}$ не вызовет никаких перемещений и управление будет передано $\mathfrak{B}_{+}^{2}$. Затем, если выходящая из $v$ дуга с меткой $e_{2}$ не принадлежит множеству $\Gamma_{\max }$, то управление будет передано автомату $\mathfrak{B}_{+}^{3}$, и т. д. Таким образом, либо в лабиринте $\tilde{L}$ будет обнаружена выходящая из $v$ дуга, принадлежащая $\Gamma_{\max }$ и тогда $\mathfrak{E}_{+}$, переместившись по этой дуге, попадет в вершину $\eta(v)$ и остановится, либо, если такой дуги не будет найдено, то после проверки максимальной выходящей из $v$ дуги (пусть ее метка будет $e_{i}$ ), то есть после окончания работы автомата $\mathfrak{B}_{-}^{i}$, управление будет опять передано $\mathfrak{B}_{-}^{i}$, в результате работы которого произойдет переход в следующую за $v$ вершину в обратной последовательности, и в этой вершине продолжится поиск выходящей дуги из множества $\Gamma_{\max }$. Таким образом, действия автомата $\mathfrak{E}_{+}$соответствуют правилу поиска вершины $\eta(v)$, а значит, автомат $\mathfrak{E}_{+}$ переместится в $\eta(v)$ и перейдет в заключительное состояние.

Рассмотрим автомат с одной краской $\mathfrak{E}_{-}$, схема которого изображена на рис. 3.

Лемма 3. Автомат $\mathfrak{E}_{-}$, будучи помещенным в начальном состоянии $q_{0}$ в произволъную вершину $v$ п-мерного прлмоугольного лабиринта $L$, переместится в вершину $\eta^{-1}(v)$ и перейдет в заключительное состояние $q_{e}$.

Доказателъство. Пусть $\tilde{L}-n$-лабиринт, порожденный $L$. В качестве доказательства приведем алгоритм нахождения вершины $\eta^{-1}(v)$ в лабиринте $\tilde{L}$, в соответствии с которым работает автомат $\mathfrak{E}_{-}$.

Переменными, изменяющимися по ходу алгоритма, будут текущая вершина $u$ и число $i$.

Шar 1. Полагаем $u:=\operatorname{Pr}_{1}\left(\gamma_{\max }(v)\right), i:=\operatorname{ind}(v)-1$. 
Шаг 2. Если $i=0$, то алгоритм останавливается, $\eta^{-1}(v)=u$. Если $i>0$, то рассматриваем выходящую из $u$ дугу с меткой $e_{i}$. Если такой дуги нет или эта дуга не принадлежит множеству $\Gamma_{\max }$, то уменьшаем $i$ на 1 и повторяем шаг 2 . В противном случае, текущей вершиной становится вершина, в которую ведет эта дуга и повторяется шаг 2.

Легко видеть, что в результате работы этого алгоритма текущей вершиной становится $\eta^{-1}(v)$, а значит, автомат $\mathfrak{E}_{-}$переместится в $\eta^{-1}(v)$ и перейдет в заключительное состояние.

Замечание 2. Автоматы $\mathfrak{E}_{+}$и $\mathfrak{E}_{-}$не изменяют раскраску лабиринта и не используют ее.

Очевидно, что автомат $\mathfrak{E}_{+}\left(\mathfrak{E}_{-}\right)$может быть изменен так, что он будет оставлять метку 1 в вершине $v$, после чего, не изменяя меток других вершин, перейдет в вершину $\eta(v)\left(\eta^{-1}(v)\right)$. Такие автоматы будем обозначать $\mathfrak{E}_{+}^{1}$ и $\mathfrak{E}_{-}^{1}$.

Предложение 3. Пусть ind $(v)=\operatorname{ind}\left(\eta^{k}(v)\right)$ и в последователъности

$$
\eta(v), \eta^{2}(v), \ldots, \eta^{k-1}(v)
$$

нет вершины с индексом, большим чем ind $(v)$. Тогда $v$ и $\eta^{k}(v)$ принадлежат одному $e_{\text {ind }(v)}-u$ uклy.

Доказателъство. Очевидно, что достаточно рассмотреть случай, когда индексы вершин

$$
\eta(v), \eta^{2}(v), \ldots, \eta^{k-1}(v)
$$

строго меньше, чем ind $(v)$. Пусть $G_{i}=\left(V_{i}, \Gamma_{i}\right)-$ компонента связности графа $G=\left(V, \Gamma_{\max }\right)$, в которую входит вершина $v$. Если дуга $\gamma_{\max }(v)$ входит в ориентированный цикл графа $G_{i}$, то дуга $\gamma_{\max }\left(\eta^{k}(v)\right)$, имеющая ту же метку, что и $\gamma_{\max }(v)$, тоже входит в этот цикл, а значит, вершины $v$ и $\eta^{k}(v)$ принадлежат одному $e_{\text {ind }(v)^{-}}$ циклу. Предположим, что $\gamma_{\max }(v)$ не входит в цикл графа $G_{i}$. Тогда $v$ является корнем некоторого поддерева одного из ориентированных от корня деревьев, присоединенных к циклу. Если бы у вершины $v$ не было выходящих дуг в графе $G_{i}$, то выполнялось бы равенство

$$
\gamma_{\max }(\eta(v))=\left(u_{j}, \eta(v)\right)
$$

для некоторой вершины $u_{j}$ из обратной последовательности для $v$, причем $\left|\left(u_{j}, \eta(v)\right)\right|>\left|\left(u_{j}, u_{j-1}\right)\right|$. Но тогда

$$
\operatorname{ind}(\eta(v))=\left|\gamma_{\max }(\eta(v))\right|>\operatorname{ind}\left(u_{j-1}\right) \geqslant \operatorname{ind}(v),
$$

что противоречит условию. Значит, $v$ имеет выходящие дуги. Пусть это будут дуги $\gamma_{1}, \gamma_{2}, \ldots, \gamma_{p}$, причем

$$
\left|\gamma_{1}\right|<\left|\gamma_{2}\right|<\ldots<\left|\gamma_{p}\right|
$$

а вершины $v_{1}, \ldots, v_{p}$ - концевые вершины этих дуг. Тогда

$$
\operatorname{ind}\left(v_{1}\right)<\ldots<\operatorname{ind}\left(v_{p}\right) \leqslant \operatorname{ind}(v) \text {. }
$$

Обход компоненты $G_{i}$, задающий перестановку $\eta$, устроен так, что сперва обходится ветвь, начинающаяся дугой $\gamma_{1}$, затем ветвь, начинающаяся дугой $\gamma_{2}$, и так далее 
до ветви, начинающейся дугой $\gamma_{p}$. Если ind $\left(v_{p}\right)<\operatorname{ind}(v)$, то индексы вершин из этих веток будут меньше, чем ind $(v)$, а значит, среди них нет вершины $\eta^{k}(v)$. В этом случае после того, как будут пройдены все вершины из поддерева с корнем $v$, следующая по обходу вершина будет такой же, как если бы у $v$ не было выходящих дуг и индекс у этой вершины был бы больше, чем у $v$, что невозможно по условию. Поэтому ind $\left(v_{p}\right)=\operatorname{ind}(v)$ и $\left|\gamma_{p}\right|=e_{\text {ind }(v)}$. Тогда первой вершиной после $v$ в порядке обхода, имеющей индекс такой же, что и $v$, будет $v_{p}$. Значит, $\eta^{k}(v)=v_{p}$. Вершина $v$ является одновременно началом дуги $\gamma_{p}$ и концом дуги $\gamma_{\max }(v)$. Следовательно,

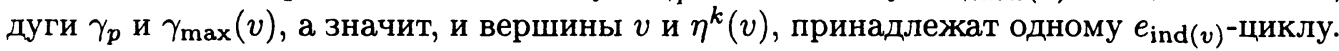

Итак, для любого $n$-лабиринта было построено разбиение множества его вершин $V$ на непересекающиеся подмножества $V_{i},\left|V_{i}\right| \geqslant 2$, и на каждом из $V_{i}$ был задан циклический порядок обхода вершин, который может быть осуществлен автоматом. В дальнейшем мы будем часто называть множества $V_{i}$ компонентами разбиения множества $V$ или просто компонентами. Будем говорить, что компонента $U$ достижима из вершины $w$ по дуге $\gamma$, если $\gamma=(w, u)$, где $u \in U$. Будем говорить, что компонента $U$ достижима из компоненты $W$ по дуге $\gamma$, если $\gamma=(w, u)$, где $w \in W$ и $u \in U$.

\section{4. Алгоритм обхода $n$-лабиринтов}

В этом параграфе будет сформулирован алгоритм обхода для порожденных $n$-лабиринтов. Основной идеей этого алгоритма будет использование множеств $V_{i}$ (компонент) как единых вершин. Такой подход позволяет в каждой такой вершине хранить информацию, достаточную для реализации обхода в глубину.

Пусть $U$ - одна из компонент, $|U|=m$. Если все вершины, входящие в $U$, имеют метки 0, то будем говорить, что компонента $U$ не помечена. Если все вершины, входящие в $U$, имеют метки 1 , то говорим, что $U$ помечена как неактивная компонента. Если $m<n+4$ и в компоненте $U$ все вершины, кроме максимальной имеют метки 1 , то говорим, что $U$ - активная компонента. Пусть $m \geqslant n+4$ и $\gamma-$ дуга, входящая в одну из вершин компоненты $U,|\gamma|=e_{k}$. Обозначим через $u_{1}, \ldots, u_{m}$ вершины этой компоненты в порядке обхода, начиная с вершины, в которую входит дуга $\gamma$. Если вершины

$$
u_{1}, u_{2}, u_{k+3}, u_{k+5}, \ldots, u_{k+1+2 i},
$$

где $i=[(m-k) / 2]-1$, имеют метки 0 , а остальные метку 1 , то говорим, что $U$ помечена как активная с входящей дугой $\gamma$, а вершину $u_{1}$ называем стартовой в этой компоненте. Заметим, что при такой разметке компоненты можно определить, используя только метки вершин, какая именно входящая дуга использовалась для разметки. Действительно, только $u_{1}$ и $u_{2}$ - две подряд идущие вершины в порядке обхода, которые имеют метки 0, а количество вершин, идущих подряд за ними и отмеченных единицей, дает индекс метки входящей дуги.

Пусть $v$ - максимальная вершина компоненты $U,|U|=m$. Упорядочим вершины, входящие в эту компоненту, пусть

$$
\eta(v)<\eta^{2}(v)<\ldots<\eta^{m}(v)=v
$$

Поскольку входящие в каждую вершину дуги упорядочены в соответствии с их метками, это позволяет упорядочить все дуги, входящие в вершины компоненты, 
а именно, если $\gamma_{1}$ и $\gamma_{2}$ таковы, что $\operatorname{Pr}_{2}\left(\gamma_{1}\right) \in U$ и $\operatorname{Pr}_{2}\left(\gamma_{2}\right) \in U$, то

$$
\gamma_{1}>\gamma_{2} \Longleftrightarrow\left(\operatorname{Pr}_{2}\left(\gamma_{1}\right)>\operatorname{Pr}_{2}\left(\gamma_{2}\right)\right) \vee\left(\operatorname{Pr}_{2}\left(\gamma_{1}\right)=\operatorname{Pr}_{2}\left(\gamma_{2}\right)\right) \&\left(\left|\gamma_{1}\right|>\left|\gamma_{2}\right|\right)
$$

Перейдем к формулировке алгоритма. Пусть $L$ - произвольный порожденный $n$-лабиринт, $v_{0}$ - начальная вершина и $U_{0}$ - компонента, содержащая $v_{0}$.

Начальный шаг. Возможны два случая.

(1) Пусть $\left|U_{0}\right|<n+4$. Размечаем $U_{0}$ как активную, в максимальной вершине выбираем выходящую дугу с минимальной меткой, перемещаемся по ней и переходим к шагу углубления.

(2) Пусть $\left|U_{0}\right| \geqslant n+4$. Выбираем дугу $\gamma_{0}$, имеющую минимальную метку среди дуг, входящих в $v_{0}$, и размечаем $U_{0}$ как активную с входящей дугой $\gamma_{0}$. После этого находим выходящую из $v_{0}$ дугу с минимальной меткой, перемещаемся по ней и переходим к шагу углубления.

Пусть $v$ - обозреваемая вершина, $\gamma$ - дуга, вдоль которой произошло последнее перемещение, $U$ - компонента, содержащая $v$.

Шаг углубления. Возможны три случая.

(1) Пусть компонента $U$ уже размечена. Возвращаемся по дуге $\gamma$ и выполняем шаг возвращения.

(2) Пусть компонента $U$ не размечена, $|U| \geqslant n+4$. Размечаем $U$ как активную с входящей дугой $\gamma$. Выбираем выходящую из $v$ дугу с минимальной меткой, перемещаемся по ней и повторяем шаг углубления.

(3) Пусть компонента $U$ не размечена, $|U|<n+4$. Проверяем, является ли $\gamma$ максимальной из дуг, по которым $U$ достижима из активных компонент. Если $\gamma$ таковой является, то размечаем $U$ как активную, в максимальной вершине выбираем выходящую дугу с минимальной меткой, перемещаемся по ней и повторяем шаг углубления. Если $\gamma$ не максимальна, то возвращаемся по $\gamma$ и выполняем шаг возвращения.

Шаг возвращения. Если $U$ - неактивная компонента, то алгоритм останавливается.

Если в вершине $v$ имеются выходящие дуги (по крайней мере одна) с метками, большими чем у $\gamma$, то выбираем среди них дугу с минимальной меткой, перемещаемся по ней и выполняем шаг углубления.

Если $\gamma$ имеет максимальную метку среди выходящих из $v$ дуг, то переходим в вершину $v^{\prime}$, следующую за $v$ в порядке обхода компоненты $U$. Далее возможны два случая.

Пусть $|U| \geqslant n+4$. Если $v^{\prime}$ является стартовой в компоненте $U$, то находим по разметке метку входящей дуги, размечаем $U$ как неактивную, перемещаемся по дуге, входящей в вершину $v^{\prime}$ и имеющей найденную ранее метку, а затем повторяем шаг возвращения. Если $v^{\prime}$ не является стартовой, то выбираем выходящую из $v^{\prime}$ дугу с минимальной меткой, перемещаемся по ней и выполняем шаг углубления. 
Пусть $|U|<n+4$. Если $v^{\prime}$ является максимальной в компоненте $U$ и $U$ достижима из активных компонент, то выбираем максимальную дугу из дуг, по которым $U$ достижима из этих компонент, предварительно разметив $U$ как неактивную, возвращаемся по этой дуге и выполняем шаг возвращения. Если $v^{\prime}$ минимальная, но нет активных компонент, из которых $U$ достижима, то алгоритм останавливается. Наконец, если $v^{\prime}$ не минимальная, то выбираем выходящую из $v^{\prime}$ дугу с минимальной меткой, перемещаемся по ней и выполняем шаг углубления.

Работу алгоритма можно описать следующим образом. В процессе работы образуется цепочка активных компонент. Для активной компоненты, отличной от начальной, предыдущая в цепочке определяется так. Если компонента помечена как активная с входящей дугой $\gamma$, то предыдущей будет компонента, которой принадлежит вершина, из которой выходит дуга $\gamma$. Если в активной компоненте меньше, чем $n+4$ вершины, и следовательно, она помечена просто как активная, то предыдущей компонентой будет та, которой принадлежит начальная вершина дуги, являющейся максимальной среди дуг, по которым данная компонента достижима из других активных компонент.

Работа всегда происходит с последней в цепочке компонентой. Обозначим ее $U$. Сперва выбирается одна из дуг (обозначим ее $\gamma$ ), выходящих из стартовой (или максимальной) вершины, и рассматривается компонента, в которую входит конечная вершина дуги $\gamma$. Проверяется, является ли эта компонента непомеченной и, когда число вершин в компоненте меньше, чем $n+4$, дополнительно проверяется, является ли дуга $\gamma$ максимальной среди дуг, по которым она достижима из активных компонент. Если проверка оказывается успешной, то эта компонента добавляется в цепочку и начинается просмотр дуг уже в этой компоненте. Если не удается добавить компоненту в цепочку, то в компоненте $U$ выбирается другая дуга, выходящая из той же вершины. Если исчерпан запас дуг из одной вершины, то начинается рассмотрение дуг, выходящих из следующей по обходу вершины компоненты $U$.

Таким образом, последовательно просматриваются все дуги, исходящие из вершин компоненты $U$. Когда все дуги будут просмотрены, компонента $U$ размечается как неактивная (и, следовательно, исключается из цепочки) и алгоритм возвращается к рассмотрению предыдущей активной компоненты. В этой компоненте возобновляется просмотр выходящих дуг, начиная с дуги, по которой произошел переход к компоненте $U$.

После того, как будут просмотрены все выходящие дуги в компоненте, содержащей начальную вершину, алгоритм останавливается, либо не обнаружив компоненты, в которую может вернуться (случай $\left|U_{0}\right|<n+4$ ), либо попав в неактивную компоненту после возвращения по фиктивной входящей дуге, выбранной на начальном шаге.

Убедимся, что в результате будут обойдены все компоненты. Пусть это не так. Тогда, в силу сильной связности $n$-лабиринта, найдется непомеченная компонента $U$, которая достижима из пройденной в процессе работы алгоритма компоненты $W$. Значит, в какой-то момент рассматривалась дуга, ведущая из $W$ в $U$. Компонента $U$ могла остаться не добавленной в цепочку только в случае, если $|U|<n+4$ и были другие активные компоненты, из которых $U$ достижима. Но тогда $U$ должна была быть включена в цепочку позднее, когда рассматривалась компонента, из которой исходит максимальная дуга, по которой достижима $U$. Поэтому, в результате работы алгоритма были рассмотрены все компоненты и размечены, в итоге, как неактивные. 
Таким образом, справедливо следующее утверждение.

Предложение 4. Описанный выше алгоритм обходит произволъный порожденный $n$-лабиринт и останавливается после обхода, пометив все вериины меткой 1 .

\section{5. Доказательство теоремы 1}

В качестве доказательства будет построен в явном виде автомат, который обходит произвольный $n$-мерный прямоугольный лабиринт и останавливается после обхода. Этот автомат будет работать в соответствии с алгоритмом, описанным в предыдущем параграфе. Будут построены автоматы, которые реализуют каждый шаг алгоритма. Для этого нам понадобятся несколько вспомогательных автоматов. Существование таких автоматов будет очевидно, поэтому приведем лишь их описание.

Сперва опишем автоматы, которые занимаются раскраской компонент.

Пусть $\mathfrak{P}_{k}^{A}, k=1, \ldots, n-$ автомат с начальным состоянием $q_{0}$ и заключительным состоянием $q_{e}$. Этот автомат, если его поместить в вершину $v$ непомеченной компоненты $U,|U| \geqslant n+4$, разметит эту компоненту как активную с входящей дугой, имеющей метку $e_{k}$, и стартовой вершиной $v$. Автомат $\mathfrak{P}_{k}^{A}$ работает следующим образом. Сначала он переходит в вершину $\eta^{-1}(v)$, оставляет в ней метку 1 и возвращается в $v$. Далее он посещает вершины компоненты $U$ в порядке обхода, задаваемом перестановкой $\eta$. При этом вершину $v$ и следующую за ней он оставляет непомеченными, затем помечает единицей подряд $k$ вершин и далее помечает единицей каждую вторую вершину до тех пор, пока не дойдет до уже помеченной вершины. Это будет вершина $\eta^{-1}(v)$. После этого он переходит в $v$ и останавливается.

Пусть $\mathfrak{P}^{M}$ - автомат с начальным состоянием $q_{0}$ и заключительным состоянием $q_{e}$. Если его поместить в максимальную вершину $v$ непомеченной компоненты $U$, $|U|<n+4$, то он разметит эту компоненту как активную и остановится в вершине $v$. Автомат $\mathfrak{P}^{M}$ работает следующим образом. Сначала он ставит метку 1 в вершину $\eta^{-1}(v)$, затем, оставив вершину $v$ непомеченной, ставит метки 1 в вершины компоненты $U$, проходя их в порядке обхода, пока не достигнет уже помеченной вершины $\eta^{-1}(v)$. После этого он переходит в $v$ и останавливается.

Пусть $\mathfrak{P}^{N}$ - автомат с начальным состоянием $q_{0}$ и заключительными состояниями $q_{e}^{1}, q_{e}^{2}, \ldots, q_{e}^{n}$. Автомат $\mathfrak{P}^{N}$, если его поместить в вершину $v$ компоненты $U$, помеченной как активная с входящей дугой $\gamma,|\gamma|=e_{i}$, то он разметит эту компоненту как неактивную и остановится в вершине $v$ в состоянии $q_{e}^{i}$. Этот автомат работает следующим образом. Он проходит вершины компоненты $U$ в порядке обратном порядку обхода, начиная с вершины $\eta^{-1}(v)$ и запоминает количество подряд идущих вершин с меткой 1. Если встречается вершина с меткой 0 , то он ставит в ней метку 1 и со следующей вершины начинает отсчет вершин, помеченных 1, заново. Если встретились подряд две непомеченные вершины, а непосредственно перед этим было подряд $k$ вершин, помеченных меткой 1 , то автомат $\mathfrak{P}^{N}$ останавливается в состоянии $q_{e}^{k}$.

Теперь опишем автоматы, которые не изменяя меток вершин, проверяют те или иные свойства текущей компоненты.

Пусть $\mathfrak{C}^{P}$ - автомат с начальным состоянием $q_{0}$ и заключительными состояниями $q_{e}^{0}$ и $q_{e}^{1}$. Этот автомат, будучи помещенным в вершину $v$ компоненты $U$, определяет является эта компонента помеченной или нет, возвращается в вершину $v$ 
и останавливается в случае положительного ответа в состоянии $q_{e}^{1}$, а в случае отрицательного ответа останавливается в состоянии $q_{e}^{0}$. Работа автомата $\mathfrak{C}^{P}$ основана на том, что, в помеченной компоненте не может быть трех подряд непомеченных вершин в порядке обхода.

Пусть $\mathfrak{C}^{A}-$ автомат с начальным состоянием $q_{0}$ и заключительными состояниями $q_{e}^{0}$ и $q_{e}^{1}$. Автомат $\mathfrak{C}^{A}$, если его поместить в вершину $v$ компоненты $U$, определяет является ли данная компонента активной, возвращается в вершину $v$ и останавливается в состоянии $q_{e}^{1}$, если $U$ - активная, и в состоянии $q_{e}^{0}$, если это не так. Для определения активности компоненты автомат $\mathfrak{C}^{A}$ использует тот факт, что в активной компоненте не может быть более двух подряд вершин, помеченных нулем, и более $(n+2)$ вершин, помеченных единицей.

Пусть $\mathfrak{C}_{i}^{j}, i, j=1, \ldots, n,-$ автомат с начальным состоянием $q_{0}$ и заключительными состояниями $q_{e}^{0}$ и $q_{e}^{1}$. Автомат $\mathfrak{C}_{i}^{j}$, будучи помещенным в вершину $v$ непомеченной компоненты $U$ такой, что $|U|<n+4$ и индекс максимальной вершины $v_{\max }$ компоненты $U$ равен $j$, перемещается в вершину $v_{\max }$ и останавливается в состоянии $q_{e}^{1}$, если Еходящая в $v$ дуга с меткой $e_{i}$ является максимальной среди дуг, по которым $U$ достижима из активных компонент, или, в противном случае, вернется в вершину $v$ и остановится в состоянии $q_{e}^{0}$. Автомат $\mathfrak{C}_{i}^{j}$ последовательно проходит вершины $v, \eta(v), \eta^{2}(v), \ldots$, пока не достигнет вершины $v_{\max }$, и в каждой вершине проверяет, имеется ли входящая дуга, идущая из активной компоненты (в вершине $v$ проверяются только входящие дуги с метками большими, чем $e_{i}$ ). Если в результате проверки этих вершин (включая $v_{\max }$ ) не найдено дуги, идущей из активной компоненты, то автомат $\mathfrak{C}_{i}^{j}$ останавливается в вершине $v_{\max }$ в состоянии $q_{e}^{1}$. Если же в какой-то момент будет найдена такая дуга, то он возвращается в вершину $v$ и переходит в состояние $q_{e}^{0}$. Максимальную вершину $v_{\max }$ автомат $\mathfrak{C}_{i}^{j}$ определяет, исходя из индекса $j$ этой вершины. Вершины с индексом $j$, входящие в компоненту $U$, образуют $e_{j}$-коридор, а вершина $v_{\max }$ является конечной вершиной этого коридора. Таким образом, $v_{\max }$ - единственная вершина в компоненте $U$, для которой $\bar{e}_{j} \in d_{\text {out }}\left(v_{\max }\right)$ и $e_{j} \notin d_{\text {out }}\left(v_{\max }\right)$.

Пусть $\mathfrak{L}-$ автомат с начальным состоянием $q_{0}$ и заключительными состояниями $q_{>}, q_{<}^{1}, q_{<}^{2}, \ldots, q_{<}^{n}$. Автомат $\mathfrak{L}$, если его поместить в вершину $v$ компоненты $U$, проверяет выполняется ли неравенство $|U| \geqslant n+4$ и, если $|U|<n+4$, то определяет индекс максимальной вершины компоненты $U$. В любом случае, он возвращается в вершину $v$ и останавливается в состоянии $q_{>}$, если $|U| \geqslant n+4$, или в состоянии $q_{<}^{i}$, если $|U|<n+4$ и $i$ - индекс максимальной вершины. Проверка мощности компоненты основана на предложении 3. Рассмотрим последовательность $v, \eta(v), \eta^{2}(v), \ldots$ Если вершины $\eta^{k}(v)$ и $\eta^{k+l}(v)$ имеют одинаковый индекс $i$ и у вершин

$$
\eta^{k+1}(v), \ldots, \eta^{k+l-1}(v)
$$

индексы не превосходят $i$, то, по предложению $3, \eta^{k}(v)$ и $\eta^{k+l}(v)$ принадлежат одному $e_{i}$-коридору. Если про $\eta^{k}(v)$ и $\eta^{k+l}(v)$ известно дополнительно, что они являются конечными вершинами $e_{i}$-коридора, то это означает, что $\eta^{k}(v)=\eta^{k+l}(v)$. Тогда мощность компоненты $U$ не превосходит $l$, а вершина $\eta^{k}(v)$ максимальна в этой компоненте. Автомат $\mathfrak{L}$ последовательно посещает вершины

$$
v, \eta(v), \eta^{2}(v), \ldots, \eta^{2 n+4}(v)
$$

и запоминает на каждом шаге максимальный индекс и была ли вершина с таким индексом конечной вершиной соответствующего коридора. Если в какой-то момент 


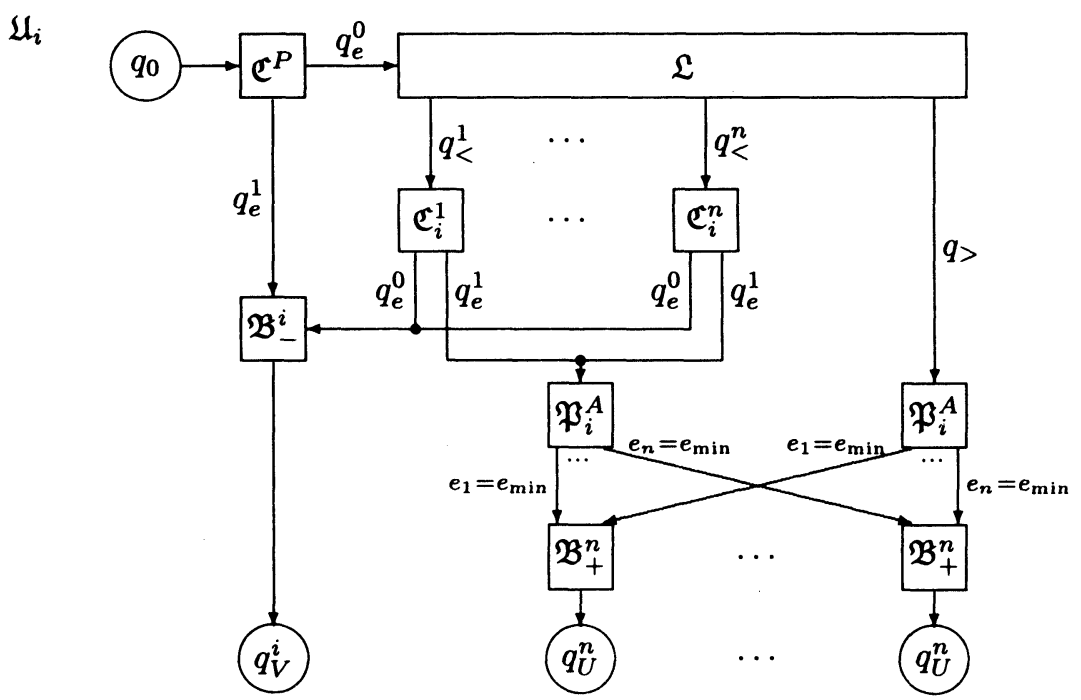

Pис. 4.

вершина с максимальным индексом, являющаяся конечной в коридоре, появляется снова, то эта вершина максимальная в компоненте $U$. Автомат запоминает ее индекс и начинает возвращаться в вершину $v$, проходя последовательность $v, \eta(v), \eta^{2}(v), \ldots$ в обратном направлении. Если максимальная вершина встретится не более, чем через $n+3$ шага, то автомат возвращается в вершину $v$ и переходит в состояние $q_{<}^{i}$, соответствующее индексу максимальной вершины. В остальных случаях автомат возвращается в вершину $v$ и переходит в состояние $q_{>}$.

Пусть $\mathfrak{S}$ - автомат с начальным состоянием $q_{0}$ и заключительными состояниями $q_{e}^{0}, q_{e}^{1}, \ldots, q_{e}^{n}$. Автомат $\mathfrak{S}$, будучи помещенным в максимальную вершину $v$ активной компоненты $U,|U|<n+4$, ставит метку 1 в вершину $v$, находит максимальную из входящих дуг, по которой $U$ достижима из активных компонент, перемещается по этой дуге и останавливается в одном из состояний $q_{e}^{1}, \ldots, q_{e}^{n}$, в зависимости от метки этой дуги (если метка $e_{i}$, то в состоянии $q_{e}^{i}$ ). Если такой дуги нет, то он останавливается в состоянии $q_{e}^{0}$.

Перейдем теперь к построению автоматов, реализующих шаги алгоритма.

Начальный шаг алгоритма является, по существу, шагом углубления, где в качестве дуги, вдоль которой произошло последнее перемещение, берется минимальная входящая в $v_{0}$ дуга.

Работа алгоритма на шаге углубления зависит от дуги, по которой произошло последнее перемещение (будем называть ее, для краткости, текущей дугой). Поэтому, мы построим $n$ автоматов $\mathfrak{U}_{i}, i=1, \ldots, n$, причем $\mathfrak{U}_{i}$ будет реализовывать шаг углубления, если его поместить в текущую вершину и метка текущей дуги равна $e_{i}$. Автомат $\mathfrak{U}_{i}$ имеет начальное состояние $q_{0}$ и заключительные состояния

$$
q_{V}^{i}, q_{U}^{1}, \ldots, q_{U}^{n}
$$

Он заканчивает свою работу в вершине, которая будет текущей на следующем шаге, заключительное состояние $q_{V}^{i}$ указывает на то, что далее должен выполняться шаг 


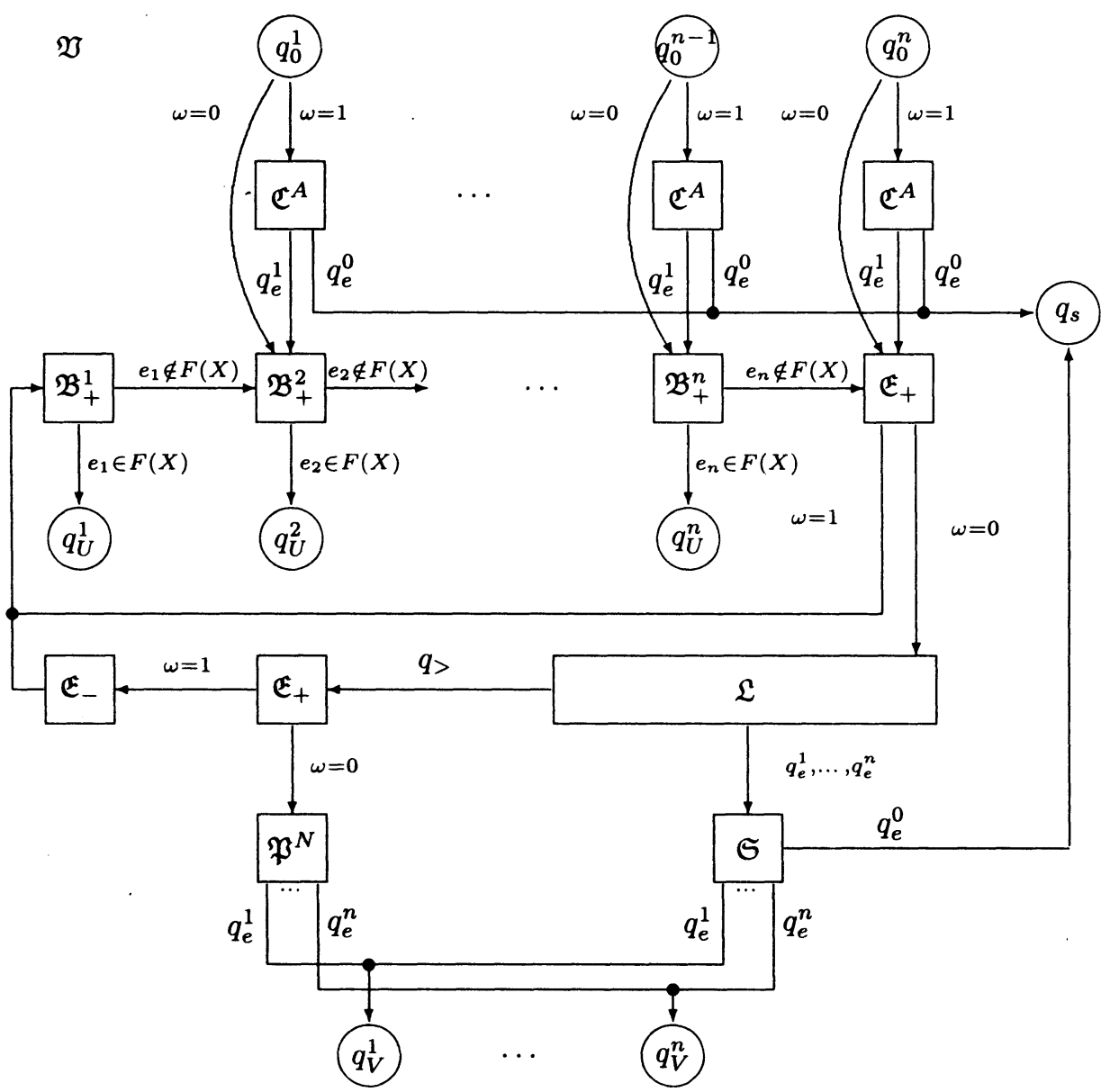

Pис. 5.

возвращения и $e_{i}$ - метка будущей текущей дуги, заключительное состояние $q_{U}^{j}$ указывает на то, что далее должен выполняться шаг углубления и $e_{j}-$ метка текущей дуги. Схема автомата $\mathfrak{U}_{i}$ изображена на рис. 4. Через $e_{\min }$ обозначена минимальная метка из множества $F(X)$.

Шаг возвращения будет реализован автоматом $\mathfrak{V}$, который имеет $n$ начальных состояний

$$
q_{0}^{1}, \ldots, q_{0}^{n}
$$

(если метка текущей дуги равна $e_{i}$, то управление передается на начальное состояние $\left.q_{0}^{i}\right)$ и заключительные состояния

$$
q_{s}, q_{U}^{1}, \ldots, q_{U}^{n}, q_{V}^{1}, \ldots, q_{V}^{n}
$$

Состояние $q_{s}$ соответствует остановке алгоритма, состояния $q_{U}^{i}\left(q_{V}^{i}\right)$ указывают на переход к шагу углубления (возвращения). Схема автомата $\mathfrak{V}$ изображена на рис. 5. 
Из автоматов $\mathfrak{V}, \mathfrak{U}_{i}, i=1, \ldots, n$, легко получаем автомат, который реализует алгоритм обхода порожденных $n$-лабиринтов. Это и есть искомый автомат.

Автор выражает искреннюю благодарность В. А. Буевичу, под руководством и при постоянной поддержке которого была написана эта статья, а также В. Б. Кудрявцеву за внимание, проявленное к данной работе.

\section{Список литературы}

1. Budach L., Automata and labyrinths. Math. Nachrichten (1978) 86, 195-282.

2. Кудрявцев В. Б., Алешин С. В., Подколзин А. С., Введение в теорию автоматов. Наука, Москва, 1985.

3. Кудрявцев В. Б., Ушчумлич Ш., Килибарда Г., О поведении автоматов в лабиринтах. Дискретная математика (1992) 4, №3, 3-28.

4. Зыричев А. Н., О синтезе автомата, обходящего плоские лабиринты с ограниченными дырами. Дискретная математика (1991) 3, №1, 105-113.

5. Золотых А. А., Обход лабиринтов с ограниченными в фиксированных направлениях дырами. Дискретная математика (1993) 5, №1, 59-69.

6. Blum M., Kozen D., On the power of the compass. Proc. 19th Annual Symposium on Foundations of Computer Sci., 1978, 132-142.

7. Hemmerling A., Normed two-plane traps for finite systems of cooperating compass. J. Inf. Process Cybern. (1987) 28, №8,9, 453-470.

8. Насыров А. З., Об обходе лабиринтов автоматами, оставляющими нестираемые метки. Дискретная математика (1997) 9, №1, 123-133.

Статья поступила 26.06.2000. 